\title{
Instant First-Person Posture Estimation
}

\author{
Takafumi Serizawa $^{1}$ and Yasuyuki Yanagida ${ }^{2}$ \\ ${ }^{1}$ Graduate School of Science \& Technology, Meijo University \\ ${ }^{2}$ Faculty of Science and Engineering, Meijo University \\ 1-501 Shiogamaguchi, Tempaku-ku, Nagoya, 468-8502 Japan \\ m0941501@ccalumni.meijo-u.ac.jp, \\ yanagida@.meijo-u.ac.jp
}

\begin{abstract}
We propose an instant posture estimation technique, which can operate only by a stereo image with small $(6 \mathrm{~cm})$ baseline. It does not require a priori information of the target user, background information, and markers. This system allows cameras to move freely, because it operates only with a small stereo camera unit. Moreover, if the input image is replaced with a movie or real-time video, this system can be used as a real-time motion tracker. With our proposed technique, robots and computers will be able to non-verbally communicate with unspecified people as well as pre-registered people. Moreover, this system can output not only the posture but also the body size and clothes. Therefore, proposed technique can also be used as a calibration procedure for other motion tracking algorithms.
\end{abstract}

Keywords: posture estimation, motion tracking, Marker-less, Calibration-free.

\section{Introduction}

The motion tracking technology is necessary to achieve a natural interaction of human and computer/robot as will as recognition of voice and facial expression. Current "motion tracking" technologies based on volume matching or image matching have achieved high-speed and high-accuracy. However, existing techniques require a priori information of the person being tracked [1]. When the target is a specific user, a priori information of the target user can be input before the tracking session. But, when the user is unspecified, it is impossible to input a priori information. To solve this problem, it is necessary to develop a new algorithm to estimate initial information based on the input of single frame.

This paper explains the "posture estimation" algorithm to acquire initial information and the entertainment application using our algorithm.

\section{Proposed Technique}

We propose a robust algorithm of posture estimation (Fig. 1), by avoiding error propagation from body trunk to ends of limbs and by using reliable 3D body ridge 
line detection in estimating the position/orientation of each part of the body. To avoid the error propagation, we introduced loose coupling of bones and a mechanism to feedback the estimation result of upper arms to the rotation angle of body trunk (Fig. 2). Unlike existing methods that use rigid joint-bone structure and estimate the direction/rotation of each part serially, estimation error in the rotation angle of body trunk does not propagate to the errors at the end of limbs (Fig. 3).
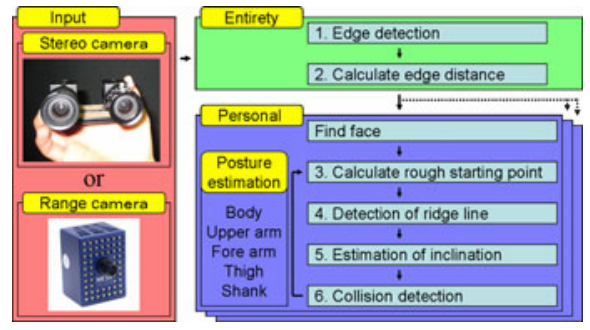

Fig. 1. System

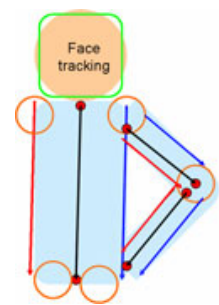

Fig. 2. Proposed

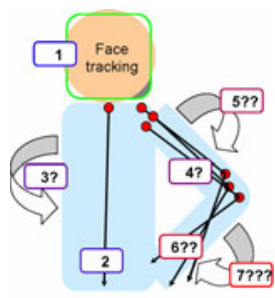

Fig. 3. Related

\section{System Performance}

Fig. 4-7 shows the case that parts of the body (lower legs) are out of the camera view, with complex background. Fig. 8-11 shows the case that entire body is visible with simple background.

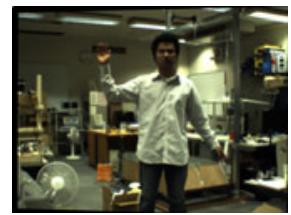

Fig. 4. Left input A

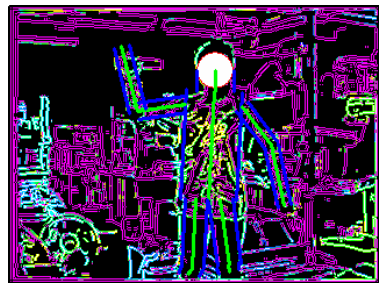

Fig. 6. 2D Output A

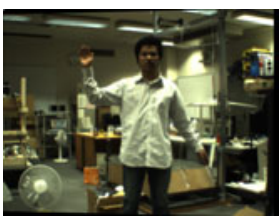

Fig. 5. Right Input A

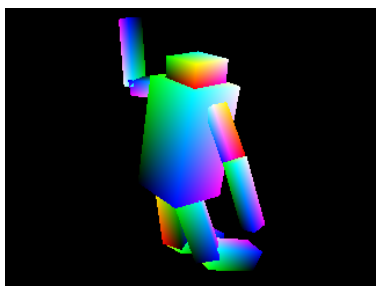

Fig. 7. 3D Output A 


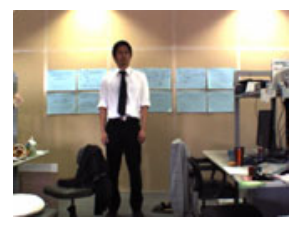

Fig. 8. Left input B

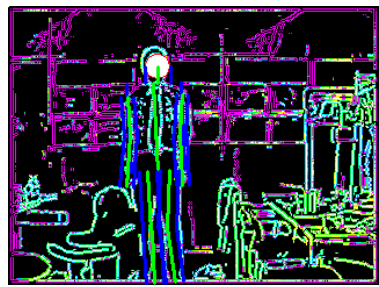

Fig. 10. 2D Output B

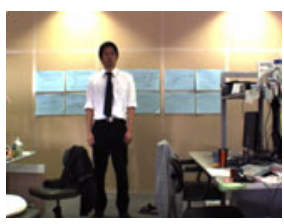

Fig. 9. Right Input B

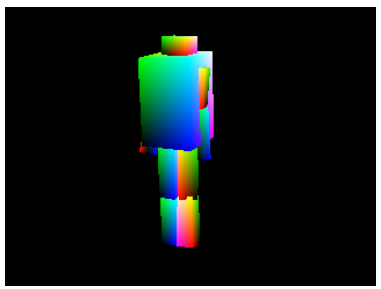

Fig. 11. 3D Output B

\section{Our Demonstration}

In our demonstrations, a visitor (A) is asked to put on a headgear composed of an HMD and a stereo camera. When another person (B) enters the visitor's view, the appearance of B is replaced by a 3D character (Fig. 12).

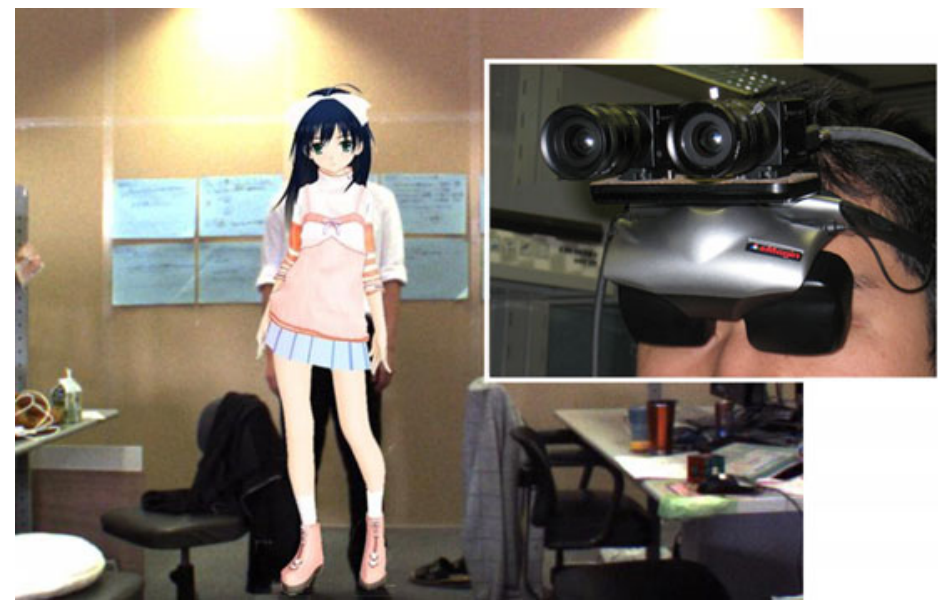

Fig. 12. Demonstration (3D model and character (C) Teatime.inc)

\section{Reference}

1. Olivier, B., Pascal, C.M.C., Arnaud, B.: Fast nonparametric belief propagation for real-time stereo articulated body tracking. Computer Vision and Image Understanding 113(1), 29-47 (2009) 